\title{
Proposition and Comparison of Catadioptric Homography Estimation Methods
}

\author{
Christophe Simler, Cédric Demonceaux, and Pascal Vasseur \\ C.R.E.A, E.A. 3299, \\ 7, rue du moulin neuf, 80000 Amiens, France \\ chris_simler@yahoo.fr, \{cedric.demonceaux, pascal.vasseur\}@u-picardie.fr
}

\begin{abstract}
Homographies are widely used in tasks like camera calibration, tracking, mosaicing or motion estimation and numerous linear and non linear methods for homography estimation have been proposed in the case of classical cameras. Recently, some works have also proved the validity of homography for catadioptric cameras but only a linear estimator has been proposed. In order to improve the estimation based on correspondence features, we suggest in this article some non linear estimators for catadioptric sensors. Catadioptric camera motion estimation from a sequence of a planar scene is the proposed application for the evaluation and the comparison of these estimation methods. Experimental results with simulated and real sequences show that non linear methods are more accurate.
\end{abstract}

Keywords: Omnidirectional Vision, Homography estimation.

\section{Introduction}

Since thirty years, many computer vision studies have been performed in order to have some information on the trajectory of a mobile perspective camera, with only the image sequence and the intrinsic parameters (calibrated camera) [1], 2, 3]. Without prior knowledge about the scene, this motion is always partially obtained because the translation is known up to a scale factor. In the case of a planar scene or a pure rotation motion or both, two images are related with a homography. From such a homography, the rotation, the direction of the translation and the direction of the normal to the plane can be computed [4]. Homographies have also other multiple applications like camera calibration [5], mosaicing [6], visual servo-control law computation in robotic [7]. Since the estimation of a homography requires data matching between two images, different kinds of primitives can be used. Thus, in 8 a dense matching based on grey level of pixels is proposed. However, this approach is iterative and a right initial value is required. Then, if the motion between the two images is too large, the method becomes inadequate and a solution consists then in performing the estimation with other kind of features such as contours [9], lines or points [10]. In this way, lines or points can be used with a linear estimator in order to provide the initial value to an iterative non linear approach which provides more stability with respect to correspondence coordinate noise [11.

D. Mery and L. Rueda (Eds.): PSIVT 2007, LNCS 4872, pp. 484 496, 2007.

(C) Springer-Verlag Berlin Heidelberg 2007 
Some recent works deal with the motion estimation with central catadioptric image sequence. Some motion and structure reconstruction methods have been proposed in 12 and in 13 . However, when the scene is planar and if only the motion is required, some less computational methods can be applied. In [14 the authors mention that the epipolar geometry is non linear between two omnidirectional images. Then, in order to recover a similar epipolar geometry as in the perspective case, the solution consists in projecting the images on the unitary sphere [15]. In this way, if two catadioptric images of a planar scene are projected on the sphere, a homography relates them and it is then possible to use a linear estimation algorithm almost similar to the one used with a perspective camera. However, the non linear estimation of a homography has not yet really been studied in the catadioptric case except in 16] where a non linear approach has been suggested based on grey level matching. However, in this case only small displacements are authorized in order to perform the iterative process. In [17, the authors also present a non linear estimation technique but only for the case of catadioptric camera calibration.

In order to test our different estimation algorithms, we consider that a catadioptric camera moves in an urban area or in an indoor scene. Such environments are generally composed of planes. In fact, we consider just a planar scene, a study with several planes will be performed in further works. We have a set of matched image points (noisy inliers) and our aim is to optimise the homography estimation process. The motion computation is then optimised because it depends directly on the homography.

In order to perform the optimisation, we suggest in this article four non linear homography estimators for catadioptric sensors. The estimations are done from matched points. Their stabilities with respect to correspondence noise are quantified and compared with the results of the catadioptric linear estimator by simulations. Some tests with matched points with real omnidirectional images validate these simulations.

We also perform some simulation tests in the perspective case by quantifying the precision of the perspective non linear estimator and by comparing it with the results of the perspective linear one. It is well known that in the pinhole case the non linear approach is better 11, but this experiment enables above all to compare the precisions of the catadioptric estimators to their homologues of the pinhole case. This paper is divided into four main parts. After introducing catadioptric projection, we derive the homography equations for catadioptric images in section 2. Then, we present in section 3 our different linear and non linear estimators. Section 4 is devoted to the evaluation and comparison of the methods. Finally, the estimators are tested on real sequences in section 5 .

\section{Perspective and Catadioptric Homography}

\subsection{The Unifying Catadioptric Projection Model}

The projection model defined in [18] covers all the catadioptric cameras with a single point of view. The catadioptric systems can be modelled with the following generic steps (fig 1): 
1. Projection of an object point $M$ of the scene in a point $M_{s}$ on the unit sphere centred on the inner focus $F$ of the mirror.

2. Non linear projection of the 3D point $M_{s}$ of the sphere with respect to the point $C$ in a $2 \mathrm{D}$ point, $m$, on a virtual plane (with the mirror parameter $\xi$ ).

3. Projection of the point $m$ of the virtual plane in a point $p$ on the image plane (with the camera intrinsic parameters and the 2 mirror parameters).

Due to the non linearity of the projection in step 2, it is difficult to model the geometrical relation between 2 images. However, by projecting them on the unit sphere (from $p$ to $M_{s}$ ) an epipolar geometry similar to the perspective case is recovered.

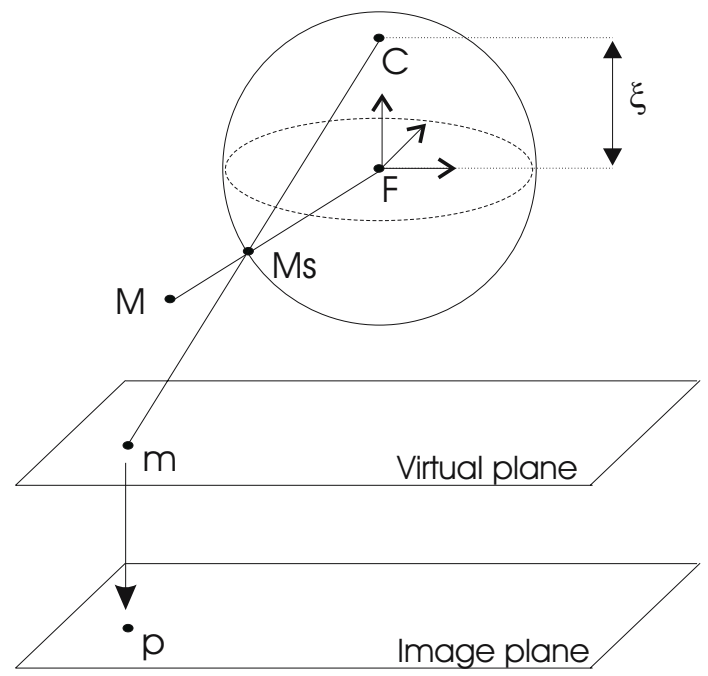

Fig. 1. Central catadioptric camera projection model

\subsection{Homography Between Two Images}

In this part we consider a couple of perspective images and another of catadioptric images. The motion between the two images is composed of rotation $R$ and translation $t$. In the two cases, an image normalisation was done. In the perspective case, the pixel coordinates were converted in meters, and in the omnidirectional case the projection on the unit sphere was done (see part 2.1). In the perspective case, the motion means the camera frame motion, and in omnidirectional it means the mirror frame motion. Figure 2 shows an illustration of these couples. $M$ is a planar scene point, and $m_{1}$ and $m_{2}$ its images before and after the motion. With the pinhole, $m_{1}=\left(x_{1}, y_{1}, 1\right)^{T}$ and $m_{2}=\left(x_{2}, y_{2}, 1\right)^{T}$ in the image frame. In the catadioptric case, $m_{1}=\left(x_{1}, y_{1}, z_{1}\right)^{T}$ and $m_{2}=\left(x_{2}, y_{2}, z_{2}\right)^{T}$ in the mirror frame. It is shown in [7] and [15] that both in perspective and omnidirectional, $m_{1}$ and $m_{2}$ are linked with a homography, called $H$, which can be written as follow : 

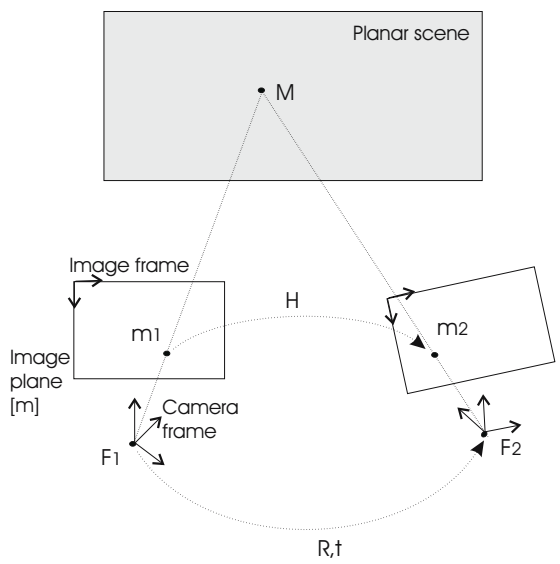

a) Perspective case: homograpy between 2 planar images.
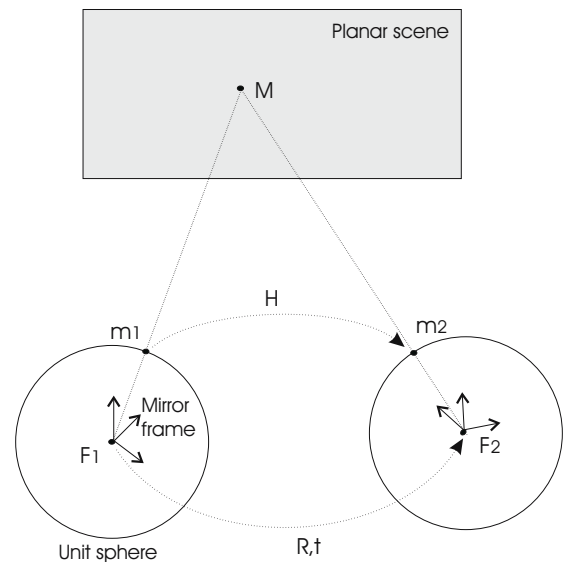

b) Catadioptric case: homography between two spherical images.

Fig. 2. Homography between perspective and catadioptric images

$$
\left(\begin{array}{l}
x_{2} \\
y_{2} \\
z_{2}
\end{array}\right)=\frac{1}{s} H\left(\begin{array}{l}
x_{1} \\
y_{1} \\
z_{1}
\end{array}\right)=\frac{1}{s}\left(\begin{array}{l}
h_{11} x_{1}+h_{12} y_{1}+h_{13} z_{1} \\
h_{21} x_{1}+h_{22} y_{1}+h_{23} z_{1} \\
h_{31} x_{1}+h_{32} y_{1}+h_{33} z_{1}
\end{array}\right)=\frac{1}{s}\left(\begin{array}{c}
H_{1}^{T} m_{1} \\
H_{2}^{T} m_{1} \\
H_{3}^{T} m_{1}
\end{array}\right)
$$

where $s$ is an unknown scale factor, $H_{i}=\left(h_{i 1}, h_{i 2}, h_{i 3}\right)^{T}$. In the pinhole case, $z_{1}=z_{2}=1 . H$ is defined up to a scale factor, thus it has only eight independent parameters. To cope with the scale factor ambiguity, we impose an arbitrary constraint, $h_{33}=1$, in order to have an unique solution.

\subsection{Motion from Homography}

Homography $H$ is expressed with motion $(R, t)$ and scene plane normal $\vec{n}$. If the norm of $\vec{n}$ is equal to the inverse of the plane initial distance, we have: $H=R+$ $t \vec{n}^{T}$. R, the direction of $t$ and $\vec{n}$ can be computed with the singular value of $H$ [4. However, this leads to 4 solutions, thus the right one was to be selected.

\section{Homography Estimators}

It has been seen in part 2 that two matched points are related with a homography $H$ in the case of a planar scene. We consider that the planar scene provides $N(N \geq 8)$ matched image points between two views. In the pinhole case, $z_{1}=z_{2}=1$. In part 3.1 we present a brief state of the art of homography estimators, and in part 3.2 we introduce the non linear ones we suggest for catadioptric cameras.

\subsection{Main Estimator Overview}

The linear estimator is currently used. Also, it exists several non linear ones for the pinhole case but since their performances are quite similar [19, we just present the most generally used. 
The linear estimator (for pinhole and catadioptric camera):

If we isolate $\mathrm{s}$ in the third equation of expression (1), we obtain:

$$
\left\{\begin{array}{l}
x_{2}=\frac{H_{1}^{T} m_{1}}{H_{3}^{T} m_{1}} z_{2} \\
y_{2}=\frac{H_{2}^{T} m_{1}}{H_{3}^{T} m_{1}} z_{2}
\end{array}\right.
$$

After linearization, the $\mathrm{i}^{\text {th }}$ correspondence provides:

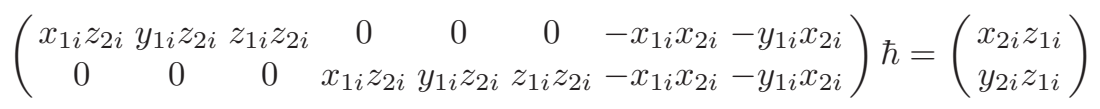

where $\hbar=\left(H_{1}^{T}, H_{2}^{T}, h_{31}, h_{32}\right)^{T}$. The expression (3) has not a strict equality because of the correspondence noise. With more than four matches it is possible to solve an overdetermined linear system of $2 \times N$ equations which can be written as follows: $A . \hbar \approx b$. The $2 N \times 8$ matrix and the $2 N$ vector $b$ are built with the correspondences. The solution obtained using the linear least squares is the following:

$$
\widehat{\hbar}=\arg \min L_{1}(\hbar)=\arg \min \sum_{i=1}^{N}\left(A_{i} \hbar-b_{i}\right)^{2}=\left(A^{T} A\right)^{-1} A^{T} b .
$$

This estimator has the advantage to provide a closed-form and unique solution. However, in the pinhole case this estimator is unstable with respect to the data noise [1]. This instability is due to the linearization of equations (2), which complicates the distributions of the error terms and thus the linear estimator (4) derives from the maximum likelihood estimator in the presence of noise. In fact, the maximum likelihood estimator is optimal because it is unbiased with minimal variance. The estimators which are close to it are generally stable, and the estimators far from it are generally unstable. In order to improve the stability of the linear estimator, a solution consists in improving the condition of the matrix $A^{T} A[2$. The techniques suggested in [2] are efficient, however they do not enable to reach the performance of the non linear estimator for pinhole camera (5) (see below). It can be noted that if the linear estimator (4) is used with a catadioptric camera, there is no need to improve the condition of $A^{T} A$. It is obvious that the projection on the unitary sphere provides automatically a low condition number.

The linear algorithm, with the pinhole as well as with the catadioptric camera is not optimal because it is not a maximum likelihood estimator. This is the reason why some non linear estimators exist for the pinhole, and why we suggest some catadioptric non linear estimators.

The non linear estimator for pinhole camera:

Let us consider expression (2) with $z_{1}=z_{2}=1$. The non linear least squares solution consists in minimising the following criterion:

$$
J_{1}(H)=\sum_{i=1}^{N}\left(x_{2 i}-\frac{H_{1 i}^{T} m_{1 i}}{H_{3 i}^{T} m_{1 i}} z_{2 i}\right)^{2}+\left(y_{2 i}-\frac{H_{2 i}^{T} m_{1 i}}{H_{3 i}^{T} m_{1 i}} z_{2 i}\right)^{2} .
$$


This function is generally minimised with the iterative algorithm of LevenbergMarquardt. This procedure needs to be initialised, and it is better to have a correct initial value in order to limit the risks of convergence toward a local minimum. The procedure is generally initialised with the linear least squares.

The advantage of this criterion with respect to the linear one is that it minimises the sum of the Euclidian reprojection errors. This means that it can be generally assumed that each error term is independent and has the same Gaussian centered distribution with respect to the exact solution. In other words it is (almost) the maximum likelihood estimator, thus it is optimal in terms of stability with respect to the noise.

\subsection{Propositions of Catadioptric Non Linear Estimators}

Our aim is to estimate with a good precision the catadioptric homography between two views, because the uncertainties of $H$ directly affect the estimated motion, which is always recovered by SVD in our work (see part 2.3). Because the linear algorithm (4) is not optimal, we suggest some catadioptric non linear estimators.

The first proposition is the minimisation of criterion (5) in the catadioptric case. However, equation (2) is not a point-to-point relation on the sphere, thus it is not the sum of the Euclidian reprojection errors which is minimised but a quantity which has no physical interpretation. In this case, nothing is known about the error term distributions. Thus, we do not know if this estimator is near of the maximum likelihood estimator (we do not know if it is stable of not).

The second proposition ensures to work with the maximum likelihood estimator. For this, we propose coming back to equation (11). The first problem with this equation is to determine the unknown scale factor $s$. We set $s=\sqrt{\left(H_{1}^{T} m_{1}\right)^{2}+\left(H_{2}^{T} m_{1}\right)^{2}+\left(H_{3}^{T} m_{1}\right)^{2}}$ because it forces $m_{2}$ to be on the unitary sphere. In this condition, we suggest minimising the sum of the Euclidian reprojection errors (proposition 2):

$$
J_{2}(H)=\sum_{i=1}^{N}\left(x_{2 i}-\frac{H_{1 i}^{T} m_{1 i}}{s_{i}}\right)^{2}+\left(y_{2 i}-\frac{H_{2 i}^{T} m_{1 i}}{s_{i}}\right)^{2}+\left(z_{2 i}-\frac{H_{3 i}^{T} m_{1 i}}{s_{i}}\right)^{2} .
$$

The properties of criterion (6) are the same as the ones of the non linear estimator for pinhole camera (see the end of part 3.1). In summary, it is optimal because it is the maximum likelihood estimator.

However, in [17] the authors suggest an estimator which applies a spherical metric to spherical images in the context of calibration. The idea is attractive because it enables to work with the metric which corresponds to our images. The adaptation of this estimator to the context of homography estimation leads us to suggest minimising the sum of the spherical reprojection errors (proposition 3):

$$
J_{3}(H)=\sum_{i=1}^{N} \arccos \left[\frac{1}{s_{i}}\left(x_{2 i} H_{1 i}^{T} m_{1 i}+y_{2 i} H_{2 i}^{T} m_{1 i}+z_{2 i} H_{3 i}^{T} m_{1 i}\right)\right] .
$$


In our opinion, this estimator is theoretically equivalent to the estimator (6), because the Euclidian reprojection error is proportional to the spherical reprojection error. It will be interesting to compare them. The drawback of criterion (7) is not its quality, but if the Levenberg-Marquardt algorithm is used to minimise it, the singularity of the derivative of arcos could be prejudicial.

To cope with this problem, two solutions are mentionned in [17, the minimisation can be done with the simplex method, or we can minimise the rope error sum (proposition 4):

$$
J_{4}(H)=\sum_{i=1}^{N}\left[2-\frac{2}{s_{i}}\left(x_{2 i} H_{1 i}^{T} m_{1 i}+y_{2 i} H_{2 i}^{T} m_{1 i}+z_{2 i} H_{3 i}^{T} m_{1 i}\right)\right]^{2} .
$$

This criterion has been introduced in 17] as the solution to solve the drawback of the previous. However, it is not the sum of the (Euclidian or spherical) reprojection errors which is minimised, thus the same remarks can be done than for the first suggested estimator $J_{1}$.

\section{Simulations}

\subsection{Simulation Conditions}

We use 3 planar patterns, containing 9, 25 and 81 points in a square of side respectively equal to 80,120 and $160 \mathrm{~m}$. These patterns are centered on the camera initial optical axis, perpendicular to this axis and situated from $100 \mathrm{~m}$ to the imaging device projection centre. The scene frame coincides with the initial camera frame. The intrinsic parameters of our pinhole are: $f=1 \mathrm{~m}$, $s_{x}=768$ pixels $/ \mathrm{m}, s_{y}=768$ pixels $/ \mathrm{m}, x_{c}=511.5$ pixels, $y_{c}=383.5$ pixels. Our catadioptric camera is composed of a parabolic mirror and an orthographic camera. The latus rectum of the parabolic mirror is equal to 2 . The actual motion between the two acquisitions is: roll $=-5^{\circ}$, pitch $=10^{\circ}$, yaw $=20^{\circ}, t_{x}=2 \mathrm{~m}$, $t_{y}=5 \mathrm{~m}, t_{z}=3 \mathrm{~m}$. With the 3 patterns and the 2 devices, we build 6 correspondence sets. A central Gaussian noise is added to the matching point coordinates. We work in fact with 5 Gaussian noises of standard deviation equal to $1 / 3,1$, $5 / 3,7 / 3,3$ pixels. The eventual outliers are rejected. The matches are then normalised (see part 2.2). After, the homography is estimated with the estimators of part 3, and the motion and the normal of the plane are computed (see part 2.3). Among the different solutions obtained by SVD, we retain the roll, pitch and yaw angles corresponding to the smallest quadratic error with the reference. Also, we retain the translation and the normal of the plane corresponding to the highest scalar product in absolute value with the reference (after normalising the vectors). The arccos of the selected scalar product provides us the angular deviation with respect to the exact direction, $\alpha_{T}$ for the translation and $\alpha_{N}$ for the normal of the plane. Finally, we are able to estimate and to select the right solution for the parameters: $\Lambda$ ( roll $=-5^{\circ}$, pitch $=10^{\circ}$, yaw $=20^{\circ}, \alpha_{T}=0^{\circ}$, $\left.\alpha_{N}=0^{\circ}\right)$. 


\subsection{Comparisons and Quantification of the Estimator Precision}

We evaluate the 7 estimators of the part 3: the linear ones for perspective and catadioptric camera, $L_{1}$, the non linear one for perspective camera, $J_{1}$, and the non linear ones for catadioptric camera (propositions 1, 2, 3 and 4 of part 3.2): $J_{1}, J_{2}, J_{3}$ and $J_{4}$. Because the homography parameters have not obvious physical interpretations, we compare the 5 parameters $\Lambda$ (roll, pitch,yaw, $\left.\alpha_{T}, \alpha_{N}\right)$. For each of the three patterns and for each of the five image point noise variances, the error on $\Lambda$ is computed with 20000 estimations as follows:

$$
\operatorname{Err}_{N, \sigma^{2}}(\Lambda)=|\operatorname{bias}(\Lambda)|+\sqrt{\operatorname{var}(\Lambda)} .
$$

In fact, $\operatorname{Err}_{N, \sigma^{2}}$ can be seen as a five components vector, each component is not a scalar but a $3 \times 5$ matrix. For each estimator, the mean values with respect to $N$ and $\sigma^{2}$ of the five components of the estimation error (4.2) are in Table 1

Table 1. Mean values of the error matrices (mean error [degree]) for each estimator

\begin{tabular}{|c|c|c|c|c|c|c|c|}
\hline & \multicolumn{2}{|c|}{ Perspective Camera } & \multicolumn{5}{|c|}{ Catadioptric Camera } \\
\hline & $L_{1}$ & $J_{1}$ & $L_{1}$ & $J_{1}$ & $J_{2}$ & $J_{3}$ & $J_{4}$ \\
\hline roll & 0.2593 & 0.2584 & 0.7077 & 0.6921 & 0.7058 & 0.7058 & 0.7398 \\
\hline pitch & 0.2541 & 0.2540 & 0.6376 & 0.6401 & 0.6382 & 0.6386 & 0.6666 \\
\hline yaw & 0.1130 & 0.1127 & 0.2720 & 0.2687 & 0.2690 & 0.2689 & 0.2845 \\
\hline$\alpha_{T}$ & 7.8027 & 7.7959 & 18.0361 & 17.9363 & 18.0032 & 18.0038 & 18.9386 \\
\hline$\alpha_{N}$ & 6.0727 & 6.0872 & 14.0271 & 13.7943 & 13.7378 & 13.7374 & 14.6840 \\
\hline
\end{tabular}

According to the presentation of part 3.2, it is assumed that the estimators $J_{2}$ and $J_{3}$ are optimal (maximum likelihood estimator) and equivalent. Thus they should be better than the linear estimator $L_{1}$ (which is not optimal, it does not minimise a physical quantity). However, it was also mentioned that we are not sure about the stabilities or the estimators $J_{1}$ and $J_{4}$.

It can be seen in Table 1 that, as predicted in part 3.2, the suggested non linear estimators $J_{2}$ and $J_{3}$ are more stable than the linear estimator $L_{1}$. Thus, it has been checked that they are nearer from the maximum likelihood estimator than $L_{1}$, this is a very encouraging result. It can also be seen in Table 1 that they present very similar results, thus the Euclidian metric is not penalizing with respect to the spherical one, and the equivalence has been checked. The small difference is due to the computation round-off errors. It can be noted that because we use the Levenberg-Marquardt algorithm to minimise each criterion, the singularity of the derivative of arcos could have deteriorated the results of $J_{3}$, but it was not the case in our experiment.

It can be seen in Table 1 that the estimator $J_{4}$ is by far the worst and even the linear estimator $L_{1}$ works better. Thus, the error term distribution is certainly very far from a Gaussian, then this estimator is far from the maximum likelihood estimator and that explains its instability. Because of the bad results obtained by $J_{4}$ in the simulations of Table 1, we consider that this estimator should not be used and we not consider it in the comparisons with real images of section 5 . 
Surprisingly, the results of the estimator $J_{1}$ seem to be as good as the results of $J_{2}$. Thus, the error term distribution is certainly close to a Gaussian, then this estimator is close to the maximum likelihood estimator and that explains its stability. That could be checked in further works. Also, an advantage of this criterion is that its simplicity may be enables a more accurate convergence in the Levenberg-Marquardt iterative minimisation process. In summary, $J_{1}, J_{2}$ and $J_{3}$ give better results than $L_{1}$ and are very similar in quality.

With the perspective camera, it can be seen in Table 1 that the non linear estimator $J_{1}$ provides some better results than the linear one $L_{1}$. It is not surprising because it is well known in the litterature [1].

It is interesting to compare the precisions of the catadioptric estimators $L_{1}$ and $J_{1}$ to their homologues of the pinhole case. According to Table 1 the perspective estimators are more precise. However, it was assumed in our simulations that the image plane of the pinhole is not limited and thus the advantage of the large field of view provided by the catadioptric camera was cancelled (in practice the huge field of view provided by a catadioptric device is sometimes essential to perform the matching between two views). In fact, what is interesting to retain about the perspective-catadioptric comparisons is that the projection of noisy data on the unitary sphere is prejudicial for the estimations.

\section{Experimental Results}

In the simulations of section 4 the seven estimators of part 3 were evaluated. We performed some simulations with perspective camera which have provided some useful additional information. However, in this part we compare only the catadioptric estimators, because it is the central point of our study. A sequence of seven images of a room is taken with a catadioptric calibrated camera (Fig. 3). The mirror frame relative attitude of each acquisition with respect to the initial acquisition is in Table 2. The homography is estimated with 18 matched points (Harris corners) belonging to a plane of the scene.

The non linear estimator $J_{4}$ is not considered because of the bad results obtained in simulation in part 4 . As it was the case with the simulations of part 4 , with real images it was also noticed that the non linear estimators $J_{1}, J_{2}$ and $J_{3}$ give some very similar results. Thus it is difficult both in simulation and with real images to separate them because their performances are very similar. In addition, with real images the imprecisions on the attitude of reference complicate the selection of an eventual best among them. In term of performance we are not able to separate them, but conceptually $J_{2}$ has some advantages: it minimises the Euclidian reprojection error sum and there is no risk of singularity by using the Levenberg-Marquardt algorithm. This is the reasons why in this section only the results of $J_{2}$ are represented and compared with the linear estimator $L_{1}$. Table 3 shows the roll, pitch, yaw errors, and the translation angular deviation between each couple of successive images using 18 matched points. Figure 4 shows the errors between each image and the first. 

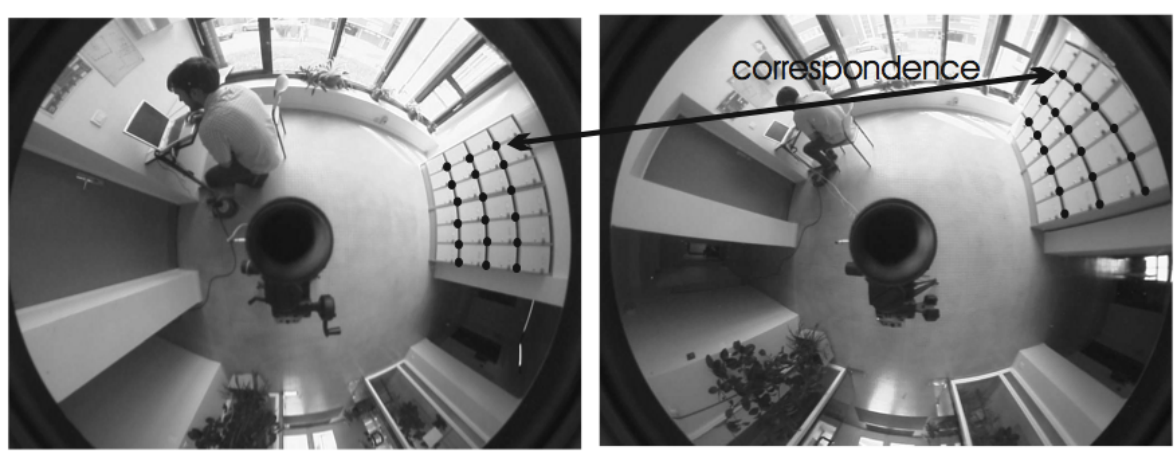

Fig. 3. Example of real scene images used in our experiments associated to the 18 matched points. The reference plane is composed of the locker on the right of the image.

Table 2. Mirror frame real attitude at each acquisition with respect to its initial position

\begin{tabular}{|c|c|c|c|c|c|c|}
\hline & Image2/1 & Image3/1 & Image4/1 & Image5/1 & Image6/1 & Image7/1 \\
\hline$t_{x}[\mathrm{~m}]$ & 0.7 & 0.7 & 0.7 & 0.7 & 0.7 & 0.7 \\
\hline$t_{y}[\mathrm{~m}]$ & 0 & 0 & 0.7 & 0.7 & 0.7 & 0.7 \\
\hline$t_{z}[\mathrm{~m}]$ & 0 & 0.1 & 0.1 & 0.1 & 0.1 & 0.1 \\
\hline roll $\left[{ }^{\circ}\right]$ & 0 & 0 & 0 & 0 & 5 & 5 \\
\hline pitch $\left[{ }^{\circ}\right]$ & 0 & 0 & 0 & 10 & 10 & 10 \\
\hline yaw $\left[{ }^{\circ}\right]$ & 0 & 0 & 0 & 0 & 0 & 10 \\
\hline
\end{tabular}

Table 3. Roll, pitch, yaw absolute errors, and translation angular deviation between each couple of successive images [degree] using 18 matched

\begin{tabular}{|c|c|c|c|c|c|c|c|c|c|c|c|c|}
\hline & \multicolumn{2}{|c|}{ Image $2 / 1$} & \multicolumn{2}{|c|}{ Image3/2 } & \multicolumn{2}{|c|}{ Image4/3 } & \multicolumn{2}{|c|}{ Image5/4 } & \multicolumn{3}{|c|}{ Image6/5 } & \multicolumn{2}{|c|}{ Image7/6 } \\
\hline & $L_{1}$ & $J_{1}$ & $L_{1}$ & $J_{1}$ & $L_{1}$ & $J_{1}$ & $L_{1}$ & $J_{1}$ & $L_{1}$ & $J_{1}$ & $L_{1}$ & $J_{1}$ \\
\hline roll & 0.7 & 0.3 & 0.1 & 0.2 & 0.2 & 0.1 & 3 & 2 & 0.3 & 0.1 & 0.9 & 0.3 \\
\hline pitch & 3 & 2 & 1.6 & 1.5 & 0.7 & 0.7 & 2 & 2 & 2 & 2 & 0.8 & 2 \\
\hline yaw & 0.3 & 1 & 0.4 & 0.1 & 0.2 & 0.2 & 0.8 & 0.7 & 0.7 & 0.4 & 0.3 & 0.5 \\
\hline$\alpha_{T}$ & 5 & 4 & 21 & 10 & 3 & 3 & 8 & 5 & 34 & 28 & 78 & 30 \\
\hline
\end{tabular}

The results show that the non linear criterion $J_{2}$ is more precise than the linear criterion $L_{1}$. Thus the results with real images are coherent with the results obtained in simulation in part 4 . However, it can be noticed in Table 3 and in figure 4 that rarely, but sometimes, $L_{1}$ provides better results than $J_{2}$. This can be explained by the fact that a poorer estimator can have a better estimate than a better one, with a probability which is low but not null in general. Also, the non linear criterion is minimised with the Levenberg-Marquardt iterative algorithm, and there is always a small risk to converge toward a local minimum. 

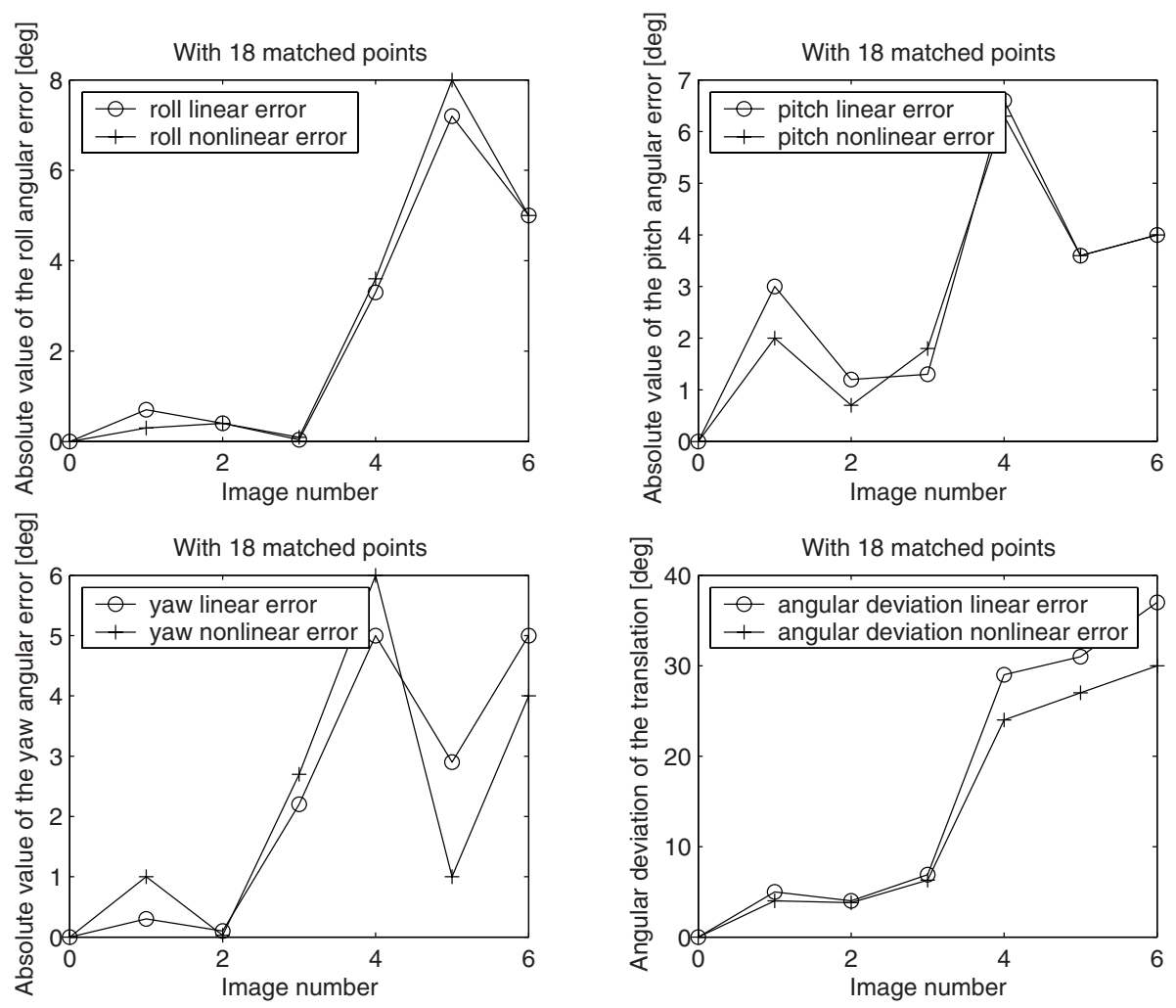

Fig. 4. Motion error between each image of the sequence and the first using 18 matched points

It can be noticed on figure 4 that the errors not always increase with respect to the image number. That is normal because a larger motion does not mean a poorer estimation. The estimation depends on the correspondences, and they are always established whatever the motion due to the large field of view of the catadioptric camera.

\section{Conclusion and Perspective}

In this paper four non linear catadioptric homography estimators were suggested and compared in a quantitative way. It has been noticed both in simulation and with real images that the performances of three of them are very similar, and above all better than the linear estimator. Our tests do not enable us to separate these three winners, but we advice to use the one called $J_{2}$ because it is the single which has these two qualities: it minimises the sum of the reprojection errors and there is no singularity problem when the minimisation is performed with the 
Levenberg-Marquardt algorithm. It is thus (almost) the maximum likelihoods estimator. The motion estimation is now optimised with a planar scene. Because we are going to work with scenes composed of several planes (urban scenes), the next step consists in optimally exploiting the different planar data sets in order to improve the motion estimation.

\section{References}

1. Longuet-Higgins, H.C.: A computer algorithm for reconstructing a scene from two projections. Nature 293(5828), 133-135 (1981)

2. Hartley, R.I.: In defense of the eight-point algorithm. IEEE Trans. Pattern Anal. Mach. Intell 19(6), 580-593 (1997)

3. Zhang, Z.: Determining the epipolar geometry and its uncertainty: A review. Int. J. Comput. Vision 27(2), 161-195 (1998)

4. Tsai, R., Huang, T., Zhu, W.: Estimating 3-d motion parameters of a rigid planar patch ii: Singular value decomposition. ASSP 30(8), 525-533 (1982)

5. Zhang, Z.: Flexible camera calibration by viewing a plane from unknown orientations. In: IIEEE Int. Conf. on Computer Vision, pp. 666-673. IEEE Computer Society Press, Los Alamitos (1999)

6. Brown, M., Lowe, D.G.: Recognising panoramas. In: IEEE Int. Conf. on Computer Vision, vol. 02, pp. 1218-1225. IEEE Computer Society, Los Alamitos (2003)

7. Benhimane, S., Malis, E.: Homography-based 2d visual servoing. In: IEEE International Conference on Robotics and Automation, IEEE Computer Society Press, Los Alamitos (2006)

8. Szeliski, R., Shum, H.Y.: Creating full view panoramic image mosaics and environment maps. In: SIGGRAPH 1997: Proceedings of the 24th annual conference on Computer graphics and interactive techniques, pp. 251-258. ACM Press/AddisonWesley Publishing Co, New York (1997)

9. Jain, P.K.: Homography estimation from planar contours. In: Third Int. Symp. on 3D Data Processing, Visualisation and Transmission, pp. 877-884 (2006)

10. Hartley, R.I., Zisserman, A.: Multiple View Geometry in Computer Vision. Cambridge University Press, Cambridge (2000)

11. Faugeras, O.: Three-dimensional computer vision: a geometric viewpoint. MIT Press, Cambridge (1993)

12. Lhuillier, M.: Automatic structure and motion using a catadioptric camera. In: Proceedings of 6th Workshop on Omnidirectional Vision OMNIVIS 2005 (2005)

13. Makadia, A., Geyer, C., Sastry, S., Daniilidis, K.: Radon-based structure from motion without correspondences. In: CVPR, pp. 796-803. IEEE Computer Society Press, Los Alamitos (2005)

14. Geyer, C., Daniilidis, K.: Mirrors in motion: Epipolar geometry and motion estimation. In: IEEE Int. Conf. on Computer Vision, pp. 766-773. IEEE Computer Society Press, Los Alamitos (2003)

15. Benhimane, S., Malis, E.: A new approach to vision-based robot control with omnidirectional cameras. In: IEEE International Conference on Robotics and Automation, IEEE Computer Society Press, Los Alamitos (2006)

16. Mei, C., Benhimane, S., Malis, E., Rives, P.: Homography-based tracking for central catadioptric cameras. In: IROS (2006) 
17. Mei, C., Rives, P.: Single view point omnidirectional camera calibration from planar grids. In: IEEE International Conference on Robotics and Automation, IEEE Computer Society Press, Los Alamitos (2007)

18. Barreto, J.: A unifying geometric representation for central projection systems. Comput. Vis. Image Underst. 103(3), 208-217 (2006)

19. Kosecka, J., Ma, Y., Sastry, S.: Optimization criteria, sensitivity and robustness of motion and structure estimation. In: ICCV 1999: Proceedings of the International Workshop on Vision Algorithms, pp. 166-182. Springer, London, UK (2000) 